\title{
Why do boys eat less fruit and vegetables than girls?
}

\author{
Elling Bere ${ }^{1,2, *}$, Johannes Brug ${ }^{3}$ and Knut-Inge Klepp ${ }^{1}$ \\ 'Department of Nutrition, University of Oslo, PO Box 1046 Blindern, 0316 Oslo, Norway: ${ }^{2}$ Department of \\ Public Health, Erasmus University Medical Centre, Rotterdam, The Netherlands: ${ }^{3} E M G O$ Institute, VU University \\ Medical Centre, Amsterdam, The Netherlands
}

\section{Submitted 5 December 2006: Accepted 15 May 2007: First published online 1 August 2007}

\begin{abstract}
Objective: To explore why boys eat less fruit and vegetables (F\&V) than girls, using longitudinal data following pupils from the age of 12.5 to 15.5 years, including perceived accessibility, modelling, intention, preferences, self-efficacy and knowledge of recommendations as potential mediators.

Design/setting/subjects: A longitudinal study, based on data collected among the control schools within the intervention project 'Fruits and Vegetables Make the Marks'. This sample contains 896 6th and 7th graders from 20 randomly selected elementary schools within two Norwegian counties. Questionnaires were administered in May 2002 and May 2005 (when the participants were in 9th and 10th grade in 18 secondary elementary schools).

Results: In single mediation analyses all determinants mediated parts of the gender difference, but only preferences decreased the gender difference to a level below statistical significance. Preferences alone explained 81\% of the gender difference. In the multiple mediation analyses, the six mediators together explained $91 \%$ of the gender difference, but only preferences and perceived accessibility contributed uniquely to the explanation, with $25 \%$ and $10 \%$ respectively.

Conclusions: Preference appears as the strongest mediator of the difference in F\&V intakes between boys and girls. Further research should explore why girls like $\mathrm{F} \& \mathrm{~V}$ more than boys.
\end{abstract}

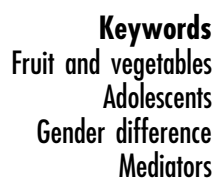

A recent comprehensive review on determinants of children's and adolescents' fruit and vegetable (F\&V) intake stated that gender is among the strongest determinants in adolescents ${ }^{1}$; in 14 of 17 reviewed European studies girls reported to eat more F\&V than boys. However, most studies assessing the relationship between gender and F\&V intake have used simple statistical methods and a cross-sectional design, not being able to assess potential interactions between gender and time. Furthermore, gender may be an important determinant of F\&V intake, but for intervention development, important and modifiable determinants need to be identified. It is therefore important to explore modifiable mediators of the gender difference in $\mathrm{F} \& \mathrm{~V}$ intake.

Only a few studies have assessed the question why males eat less F\&V than females. In a study of older adults it was reported that men's poorer nutritional knowledge explained about half of the variation in F\&V intake associated with gender, while preferences, attitudes or dieting status did not explain any of the variance ${ }^{2}$. In a study of young adults, health beliefs explained around 40\%, and dieting status $7 \%$, of the gender difference in fruit intake ${ }^{3}$. No studies, as far as we know, have assessed similar gender differences among children or adolescents. It is important to understand why boys eat less F\&V than girls in order to tailor $\mathrm{F} \& \mathrm{~V}$ promotion interventions to gender.

In earlier Norwegian studies, a number of personal modifiable determinants of F\&V intake have been identified, such as perceived accessibility of F\&V at home, modelling, intention to eat 5-a-day, preferences for $\mathrm{F} \& \mathrm{~V}$, self-efficacy to eat 5-a-day and knowledge of recommended intake levels ${ }^{4,5}$. Few studies have investigated gender differences in such determinants of adolescents' $\mathrm{F} \& \mathrm{~V}$ intake.

The aim of the present study was to explore if such factors mediate differences in F\&V intake levels between boys and girls, using longitudinal data, following pupils from the age of 12.5 to 15.5 years. A secondary aim was to assess if gender differences in intake levels changed over time.

\section{Method}

This study is part of the 'Fruits and Vegetables Make the Marks' (FVMM) project. FVMM is an intervention project including 38 randomly selected elementary schools in 
two Norwegian counties. The pupils within in the 20 control schools constitute the study sample of the present study. Data from survey questionnaires completed in May 2002 and May 2005 were used for the analyses. The questionnaire surveys were completed by the pupils in the classroom, in the presence of a trained project worker, within one school lesson (45 minutes). Research clearance was obtained from The Norwegian Social Science Data Services. The FVMM cohort includes 896 control pupils (84\%), of which 813 and 728 respectively participated in the May 2002 (mean age 12.5 years) and May 2005 (mean age 15.5 years) surveys and also reported same gender at all occasions.

F\&V intake was measured by four frequency questions: 'How often do you eat...' (1) vegetables for dinner; (2) other vegetables (e.g. carrot for school lunch); (3) apple, orange, pear or banana; (4) other fruits or berries. All four questions had 10 response alternatives ranging from 'Never' $=0$ to 'Several times a day' $=10$, giving a scale ranging from 0 to 40 times per week. The test-retest (14 days in between) correlation of this scale in a sample of 114 6th grade pupils was $0.75^{6}$. The correlation between the scale and a validation method (7-day food diaries) was 0.32 in a separate validation study of 85 6th grade pupils, which is similar to the results found in other studies of the same age group ${ }^{6}$. Most scales of the potential mediators - accessibility of F\&V at home, modelling, intention to eat 5-a-day, preferences for F\&V and selfefficacy to eat 5-a-day - included one to five statements with response alternatives ranging from 'I fully disagree' to 'I fully agree' (see reference 5 for details). These scales have been analysed for reliability, with test-retest correlations ranging from 0.51 (intention) to 0.74 (preferences) ${ }^{7}$. Knowledge (awareness of the 5-a-day recommendation) was measured by one question: 'How many servings of fruit and vegetables should a person your age eat every day?' This question had seven response alternatives ranging from 'None' to 'More than 5 a day'. Missing values on any items were substituted by the mean value for the remaining group on the respective item. To achieve a score on a scale, more than $50 \%$ of the scale items had to be answered. A total of 85 and 15 children had one or more missing values substituted, respectively, in 2002 and 2005.

A variable functions as a mediator when it meets the following conditions (see Fig. 1): (1) variations in levels of the independent variable (gender) significantly account for variations in the presumed mediator (i.e. Path a); (2) variations in the mediator significantly account for variations in the dependent variable (i.e. Path b); and (3) when Paths $\mathrm{a}$ and $\mathrm{b}$ are controlled, a previously significant relationship between the independent and dependent variables is no longer significant, with the strongest demonstration of mediation occurring when Path c is zero 8.

All analyses conducted were different mixed models of repeated measures (i.e. both 2002 and 2005 data were included in the same analyses), all adjusting for school as a random effect and time (survey), using SPSS version 14. First, gender was regressed on F\&V intake (Path c unadjusted, $\left.Y_{\mathrm{F} \& \mathrm{~V}}=\tau X_{\mathrm{SEX}}\right)$ and on the individual potential mediators independently ( $Y_{\mathrm{M}_{i}}=\tau X_{\mathrm{SEX}}$, Path a), in order to state gender differences in the respective variables (Table 1). Interaction between gender and time (2002 vs. 2005) was checked, and results were stratified on time if interaction was statistically significant (secondary research question). Since all potential mediators included in this study have been reported to correlate significantly with F\&V intake (Path b, Pearson's $r$ were 0.24-0.45 ${ }^{4}$ ), these analyses were not repeated in the present study.

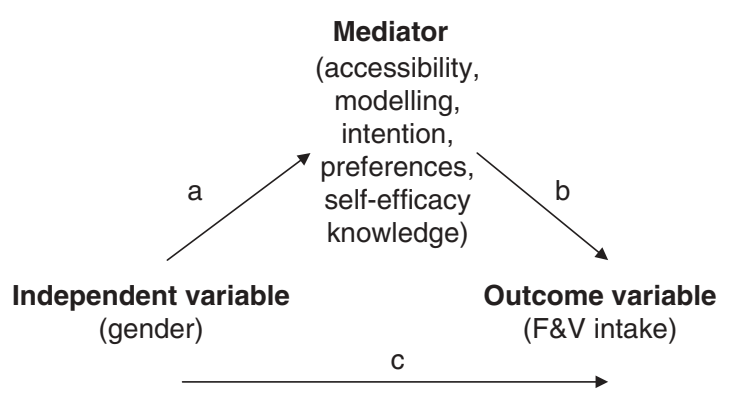

Fig. 1 Mediational model

Table 1 Gender differences in F\&V intake and indeterminants of intake

\begin{tabular}{|c|c|c|c|c|c|c|c|c|c|c|c|c|c|c|}
\hline & \multirow[b]{2}{*}{ Items in scale } & \multirow[b]{2}{*}{ Range } & \multicolumn{4}{|c|}{ Both times } & \multicolumn{4}{|c|}{2002} & \multicolumn{4}{|c|}{2005} \\
\hline & & & Boys & Girls & Difference & $P$-value & Boys & Girls & Difference & $P$-value & Boys & Girls & Difference & $P$-value \\
\hline $\mathrm{F} \& \mathrm{~V}$ intake* & 4 & $0 / 40$ & 11.9 & 14.5 & 2.6 & $\leq 0.001$ & & & & & & & & \\
\hline Accessibility & 5 & $-10 / 10$ & 3.6 & 4.9 & 1.4 & $\leq 0.001$ & & & & & & & & \\
\hline Modelling & 4 & $-8 / 8$ & 2.5 & 3.1 & 0.7 & $\leq 0.001$ & & & & & & & & \\
\hline Intentiont & 1 & $-2 / 2$ & & & & & -0.1 & 0.2 & 0.3 & 0.003 & 0.2 & 0.7 & 0.5 & $\leq 0.001$ \\
\hline Preferences $\ddagger$ & 4 & $-8 / 8$ & & & & & 1.3 & 2.9 & 1.5 & $\leq 0.001$ & 0.6 & 3.3 & 2.7 & $\leq 0.001$ \\
\hline Self-efficacy & 3 & $-14 / 14$ & 0.1 & 0.8 & 0.7 & $\leq 0.001$ & & & & & & & & \\
\hline Knowledge & 1 & $0 / 6$ & 3.5 & 3.7 & 0.2 & 0.008 & & & & & & & & \\
\hline
\end{tabular}

F\&V - fruit and vegetable.

${ }^{*}$ Difference in F\&V intake $(\tau=2.6)$.

+ Gender $\times$ time interaction: $P=0.04$.

$\ddagger$ Gender $\times$ time interaction: $P \leq 0.001$. 
Then, gender was regressed on F\&V intake adjusting for single potential mediators individually (Path c adjusted for single mediators, $Y_{\mathrm{FV}}=\tau^{\prime} X_{\mathrm{SEX}}+\beta^{\prime} X_{\mathrm{M}_{i}}$ ) (models I, Table 2) and all six potential mediators together (Path $\mathrm{C}$ adjusted for all mediators, $Y_{\mathrm{FV}}=\tau^{\prime \prime} X_{\mathrm{SEX}}+\beta^{\prime \prime} X_{\mathrm{M}_{1-6}}$ ) (model II, Table 3). This last analysis was repeated excluding each potential mediator at a time (models III, Table 3) in order to state each mediator's unique contribution to the explanation of the variance in the gender difference in F\&V intake. The proportions mediated by the mediators were calculated by subtracting the adjusted relationship between gender and F\&V intake (e.g. $\tau^{\prime}$ ) from the unadjusted $(\tau)$, and dividing the result by the unadjusted value (i.e. $\left.\left(\tau-\tau^{\prime}\right) / \tau\right)^{9}$. By subtracting the proportion mediated in models III from the proportion mediated in model II, the unique contribution to the multiple moderator model (model 2) by each moderator was calculated. An examination of the residuals did not reveal unacceptable departures from normality.

Table 2 Single mediator analyses: effect of gender on F\&V intake after adjusting for accessibility, modelling, intention, preferences, self-efficacy or knowledge

\begin{tabular}{|c|c|c|c|c|c|}
\hline & \multicolumn{5}{|c|}{ Models I } \\
\hline & \multicolumn{2}{|c|}{ Potential mediator } & \multicolumn{2}{|c|}{ Gender } & \multirow{2}{*}{$\begin{array}{c}\text { Mediated' } \\
\left(\tau-\tau^{\prime}\right) / \tau\end{array}$} \\
\hline & $\beta^{\prime}$ & $P_{\beta^{\prime}}$ & $\tau^{\prime}$ & $P_{\tau^{\prime}}$ & \\
\hline Accessibility & 0.9 & $\leq 0.001$ & 1.3 & $\leq 0.001$ & 0.49 \\
\hline Modeling & 0.8 & $\leq 0.001$ & 2.1 & $\leq 0.001$ & 0.20 \\
\hline Intention & 1.8 & $\leq 0.001$ & 1.8 & $\leq 0.001$ & 0.31 \\
\hline Preferences & 0.9 & $\leq 0.001$ & 0.5 & 0.19 & 0.81 \\
\hline Self-efficacy & 1.1 & $\leq 0.001$ & 1.8 & $\leq 0.001$ & 0.30 \\
\hline Knowledge & 1.1 & $\leq 0.001$ & 2.3 & $\leq 0.001$ & 0.10 \\
\hline
\end{tabular}

F\&V - fruit and vegitable; $\tau^{\prime}$ - difference in F\&V intake between boys and girls, while adjusting for single potential mediators; $\beta^{\prime}$ - difference in the single potential mediators between boys and girls, while adjusting for F\&V intake; mediated' - proportion of the gender difference in fruit and vegetable intake mediated by the respective factors.

\section{Results}

Table 1 shows that girls reported eating fruit and vegetables more often than boys (14.5 vs. 11.9 times per week). Girls also reported significantly higher values for all of the determinants assessed. Interactions between gender and time were seen only for intention and preferences. In both, the differences between the genders were greater in 2005 than in 2002 .

In the single mediation analyses all determinants mediated parts of the gender differences, but only preferences decreased the gender difference to a level below statistical significance (Table 2). Preferences alone explained $81 \%$ of the gender difference. In the multiple mediation analyses (Table 3), the six mediators together explained 91\% of the gender difference, with preferences contributing with the largest amount (25\%). In addition, perceived accessibility contributed with $10 \%$ of the explanation. The other factors did not contribute much to the explanation in the multiple mediator model, and most of the explanation of the variance was explained by shared variance of two or more of the six independent variables.

\section{Discussion}

The results from the present study indicate that the main reason why boys eat less $\mathrm{F} \& \mathrm{~V}$ than girls is because they like them less. In addition, differences in perceived accessibility of F\&V at home also mediated some of the variance in the gender difference. That girls have greater knowledge, and stronger intentions, self-efficacy and role models, does not appear to explain the gender-F\&V relationship when adjusting for preferences and perceived accessibility.

It has previously been reported that boys like $F \& V$ less than girls ${ }^{10}$. Even among 4-year-olds, girls have been

Table 3 Multiple mediation analyses: effect of gender on F\&V intake after adjusting for accessibility, modelling, intention, preferences, self-efficacy and knowledge (model II), and after exclusion of single factors (models III)

\begin{tabular}{|c|c|c|c|c|c|c|c|}
\hline & \multicolumn{3}{|c|}{ Model II } & \multicolumn{3}{|c|}{ Models III } & \multirow[b]{2}{*}{ Unique contr. } \\
\hline & Estimate & $P$-value & Mediated" & Estimate & $P$-value & Mediated"' & \\
\hline & $\begin{array}{l}\tau^{\prime \prime} \\
0.2\end{array}$ & $\begin{array}{c}P_{\tau^{\prime}} \\
0.52\end{array}$ & $\begin{array}{c}\left(\tau-\tau^{\prime \prime}\right) / \tau \\
0.91\end{array}$ & & & & \\
\hline \multicolumn{8}{|l|}{ Gender } \\
\hline Accessibility & 0.5 & $\leq 0.001$ & & 0.5 & 0.19 & 0.81 & 0.10 \\
\hline Modellling & 0.2 & 0.02 & & 0.2 & 0.50 & 0.91 & 0.00 \\
\hline Intention & 0.0 & 0.91 & & 0.2 & 0.51 & 0.91 & 0.00 \\
\hline Preferences & 0.5 & $\leq 0.001$ & & 0.9 & 0.01 & 0.66 & 0.25 \\
\hline Self-efficacy & 0.4 & $\leq 0.001$ & & 0.1 & 0.71 & 0.95 & -0.04 \\
\hline Knowledge & 0.4 & $\leq 0.001$ & & 0.3 & 0.47 & 0.90 & 0.01 \\
\hline
\end{tabular}

F\&V - fruit and vegitable; $\tau^{\prime \prime}$ - difference in F\&V intake between boys and girls, while adjusting for single potential mediators; $\beta^{\prime \prime}-$ difference in the single potential mediators between boys and girls, while adjusting for F\&V intake and also the other potetial mediators; $\tau^{\prime \prime \prime}-$ difference in F\&V intake between boys and girls, while adjusting for multiple potemtial mediators, not including the one in the respective row; mediated" - proportion of the gender difference in F\&V intake mediated by all determinants; except the one in the respective row; unique contribution - proportion of mediated"' uniquely explained by the respective factor. 
reported to like vegetables better than boys ${ }^{11}$. However, the question why boys like F\&V less than girls remains unanswered. It has been suggested that social desirability may have a stronger impact on girls responding to the questionnaire because of the greater importance that females attach to diet $^{3}$. A second reason could be that boys' greater liking for more energy-dense foods (i.e. not $\mathrm{F} \& \mathrm{~V}$ ) is an adaptation to their greater energy requirement $^{10}$. This hypothesis points towards physiological differences between boys and girls. Could it be that boys like F\&V less than girls mainly because boys and girls are different physiologically? In traditional hunter-gatherer societies men were hunters and women were gatherers ${ }^{12}$, and even if the food was divided between the genders, men probably ate more hunted animal food and women probably ate more gathered plants. The result might be that a difference in food preferences between males and females evolved. However, this is a hypothesis that needs to be tested.

The results from this study contradict results from a previous study that reported preferences not to mediate the gender difference among older adults' $F \& V$ intake ${ }^{2}$. Hypothetical explanations for this contradiction might be the different age groups, or the different methods used to assess both $F \& V$ intake and preferences in the two different studies.

We observed a gender difference in perceived accessibility at home, and girls scored higher than boys. However, parents within the FVMM cohort do not report the accessibility to be different for boys or girls (on a similar accessibility scale as in the present study, boys' parents scored 5.2 and girls parents scored 5.3, $P=0.62$, unpublished data; see reference 7 for scale details). The gender difference must therefore be due to how the children and adolescents perceive the accessibility and not to the accessibility at home itself. Therefore part of the gender difference in $\mathrm{F} \& \mathrm{~V}$ intake is due to the fact that girls perceive the accessibility at home to be higher, even if it probably is not true. This could also be because social desirability may have a stronger impact on girls' responses to the questionnaire ${ }^{3}$.

A limitation of the present study is the problems with co-variation. In the separate single mediator analyses all factors explained between $10 \%$ and $81 \%$ of the variance of the gender difference in $\mathrm{F} \& \mathrm{~V}$ intake. However, in the multiple mediator analyses, the effect of modelling, selfefficacy, intention and knowledge disappeared. This is because of their correlation with the other factors. Large inter-correlation between the independent variables makes it difficult to assess which variables predict behaviour and by how much ${ }^{13}$. In the multiple mediator model $91 \%$ of the variance in the gender difference in F\&V intake was explained. Preferences explained 25\% and perceived accessibility $10 \%$, but most of the remaining explained variance was shared variance by two or more factors, and it is not possible to give any one factor the credit. This is a common problem, as variables related to the same behaviour naturally correlate with one another ${ }^{4}$.

Girls scored higher than boys for both F\&V intake and for all the personal factors. For F\&V intake and for most personal factors no significant changes in the gender differences were observed over the time period. For preferences and intention the gender difference increased from 2002 to 2005.

\section{Conclusion}

Preference appears as the strongest mediator of the relationship between gender and $F \& V$ intake among adolescents. A next research step will be to assess why girls like fruit and vegetables more than boys.

\section{Acknowledgements}

Sources of funding: This study was funded by the Norwegian Research Council. The study was initiated and analysed by the investigators.

Conflict of interest declaration: The authors declare that they have no competing interest.

Authorship responsibilities: K.-I.K. conceived the FVMM project. E.B. and J.B. designed the present study and interpreted the data. E.B. analysed the data and drafted the manuscript, and J.B. and K.-I.K. revised it critically. All authors have approved the final version of the manuscript.

\section{References}

1 Rasmussen M, Krølner R, Klepp K-I, Lytle L, Brug J, Bere E, et al. Determinants of fruit and vegetable consumption among children and adolescents: a review of the literature. Part I: quantitative studies. International Journal of Behavioral Nutrition and Physical Activity 2006; 3: 22.

2 Baker AH, Wardle J. Sex differences in fruit and vegetable intake in older adults. Appetite 2003; 40: 269-75.

3 Wardle J, Haase AM, Steptoe A, Nillapun M, Jonwutiwes K, Bellisle F. Gender differences in food choice: the contribution of health beliefs and dieting. Annals of Behavioral Medicine 2004; 27: 107-16.

4 Bere E, Klepp K-I. Correlates of fruit and vegetable intake among Norwegian schoolchildren: parental and selfreports. Public Health Nutrition 2004; 7: 991-8.

5 Bere E, Klepp K-I. Changes in accessibility and preferences predict children's future fruit and vegetable intake. International Journal of Behavioral Nutrition and Physical Activity 2005; 2: 15.

6 Andersen LF, Bere E, Kolbjørnsen N, Klepp K-I. Validity and reproducibility of self-reported intake of fruit and vegetable among 6th graders. European Journal of Clinical Nutrition 2004; 58: 771-7.

7 Bere E, Klepp K-I. Reliability of parental and self-reported determinants of fruit and vegetable intake among 6th graders. Public Health Nutrition 2004; 7: 353-6. 
8 Baron RM, Kenny DA. The moderator-mediator variable distinction in social psychological research: conceptual, strategic, and statistical considerations. Journal of Personality and Social Psychology 1986; 51: 1173-82.

9 Mackinnon DP. Analysis of mediating variables in prevention and intervention research. NIDA Research Monograph 1994; 139: 127-53.

10 Cooke LJ, Wardle J. Age and gender differences in children's food preferences. British Journal of Nutrition 2005; 93: 741-6.
11 Wardle J, Sanderson S, Leigh GE, Rapoport L. Factoranalytic structure of food preferences in four-year-old children in the UK. Appetite 2001; 37: 217-23.

12 Kaplan H, Hill K, Lancaster J, Hurtado AM. A theory of human life history evolution: diet, intelligence, and longevity. Evolutionary Anthropology 2000; 9: 156-85.

13 Tabachnick BG, Fidell LS. Multiple regression. In: Tabachnick BG, Fiedell LS, eds. Using Multivariate Statistics, 4th ed. Needham Heights, MA: Allyn \& Bacon, 2001; $111-76$. 